\title{
Screening for Lynch Syndrome in Young Colorectal Cancer Patients from Saudi Arabia Using Microsatellite Instability as the Initial Test
}

\author{
Masood Alqahtani, ${ }^{1,2}$, Fabienne Grieu³, Amerigo Carrello³, Benhur Amanuel, \\ Miral Mashour ${ }^{2}$, Rabab Alattas², Khalid Al-Saleh ${ }^{4}$,Abdulmalik Alsheikh4, Sarah \\ Alqahtani $^{5}$, Barry Iacopetta ${ }^{1 *}$
}

\begin{abstract}
Background: Lynch Syndrome (LS) is a familial cancer condition caused by germline mutations in DNA mismatch repair genes. Individuals with LS have a greatly increased risk of developing colorectal cancer (CRC) and it is therefore important to identify mutation carriers so they can undergo regular surveillance. Tumor DNA from LS patients characteristically shows microsatellite instability (MSI). Our aim here was to screen young CRC patients for MSI as a first step in the identification of unrecognized cases of LS in the Saudi population. Materials and Methods: Archival tumor tissue was obtained from $284 \mathrm{CRC}$ patients treated at 4 institutes in Dammam and Riyadh between 2006 and 2015 and aged less than 60 years at diagnosis. MSI screening was performed using the BAT-26 microsatellite marker and positive cases confirmed using the pentaplex MSI analysis system. Positive cases were screened for BRAF mutations to exclude sporadic CRC and were evaluated for loss of expression of 4 DNA mismatch repair proteins using immunohistochemistry. Results: MSI was found in 33/284 (11.6\%) cases, of which only one showed a BRAF mutation. Saudi MSI cases showed similar instability in the BAT-26 and BAT-25 markers to Australian MSI cases, but significantly lower frequencies of instability in 3 other microsatellite markers. Conclusions: MSI screening of young Saudi CRC patients reveals that approximately 1 in 9 are candidates for LS. Patients with MSI are strongly recommended to undergo genetic counselling and germline mutation testing for LS. Other affected family members can then be identified and offered regular surveillance for early detection of LS-associated cancers.
\end{abstract}

Keywords: Colorectal cancer - microsatellite instability - Lynch syndrome - screening - Saudi Arabia

Asian Pac J Cancer Prev, 17 (4), 1917-1923

\section{Introduction}

Hereditary nonpolyposis colorectal cancer (HNPCC), more commonly known as Lynch syndrome (LS), is an autosomal dominant genetic condition associated with a high risk of colorectal cancer (CRC) (Lynch et al., 2003). Approximately 1-2\% of all CRC are thought to be due to LS. In addition to CRC, LS is also associated with increased risk for endometrial, gastric, ovarian, small bowel, and other rarer cancer types. LS is caused by germline mutations in DNA mismatch repair (MMR) genes, with the most frequent being MLH1, MSH2, MSH6, and PMS2. The incidence of MMR gene mutation carriers is about 1 in 500 in Western populations. Identification of these individuals is crucial because it allows them to undergo early and regular surveillance for cancer. It also allows their extended family to be screened for additional mutation carriers. Regular screening by colonoscopy has been shown to reduce mortality from CRC in mutation carriers (Jarvinen et al., 2000). Until recently, CRC patients who were suspected of being at risk for LS were identified through the use of clinical criteria which rely on obtaining a detailed family history of cancer, as outlined in the Amsterdam and Bethesda guidelines (Umar et al., 2004). However, these guidelines have been associated with low sensitivity for the detection of LS and their implementation in routine clinical practice has been poor (Lynch et al., 2004). Consequently, there have been calls to introduce laboratory-based screening tests for LS that do not require the clinician to obtain a detailed family history of cancer (Terdiman, 2005).

Tumors that arise in patients with LS have a defective

${ }^{1}$ School of Surgery, University of Western Australia, ${ }^{3}$ Department of Anatomical Pathology, Pathwest Laboratory Medicine, Queen Elizabeth II Medical Centre, Perth, Australia, ${ }^{2}$ Department of Pathology and Laboratory Medicine, King Fahad Specialist HospitalDammam, ${ }^{4}$ College of Medicine, Anatomical Pathology, Adult Oncology, King Khaled University Hospital King Saud University, ${ }^{5}$ Primary Health Care Department of Eastern Province, Ministry of Health, Riyadh, Saudi Arabia *For correspondence: barry. iacopetta@uwa.edu.au 
DNA MMR system, resulting in ubiquitous small deletions in DNA repeat regions that are referred to as microsatellite instability (MSI) (Iacopetta et al., 2010). In addition to MSI, these tumors almost always show loss of expression of one or more MMR proteins as seen with immunohistochemistry (IHC) (Marcus et al., 2009). MSI and IHC tests are therefore essential for the selection of CRC patients to undergo further germline mutation testing for LS. However, a positive MSI test and loss of MMR expression by IHC are also observed in approximately $10 \%$ of sporadic CRC, meaning they are not specific markers for the presence of LS. Fortuitously, sporadic MSI CRC cases often display a hotspot mutation in the BRAF oncogene, whereas MSI CRC from LS patients never show mutations in this oncogene. The presence of BRAF mutation can therefore be used to identify MSI CRC cases that are sporadic in origin and can thus be excluded from further testing for possible germline mutations (Domingo et al., 2004).

We previously reported the results of MSI testing in young CRC patients as a first screen to detect LS in the state of Western Australia (WA) (Schofield et al., 2009). This laboratory-based screening program was conducted in the absence of any information on the family history of cancer. It was established that initial screening for MSI, followed by testing for BRAF mutation in positive cases, was an effective strategy to identify LS mutation carriers in the WA population. Routine MSI and IHC testing was subsequently implemented for all CRC patients aged $<60$ years in WA starting in 2008. The rate of diagnosed LS cases in this state (population 2.5 million) has since increased from 2-3 per year prior to routine MSI screening to an average of 8 cases per year over the past 7 years (Schofield et al., 2014).

In Saudi Arabia (SA), CRC is the most frequent cancer type in males (13\% of all cancer cases) and the second most common cancer in females $(9 \%)$, with a total of almost 1,200 cases reported in 2011 (Al-Madouj et al., 2011). Interestingly, the mean age at diagnosis (55-58 years) is approximately 12-15 years younger compared to Western populations (Aljebreen, 2007; Mosli et al., 2012; Amin et al., 2012). Compared to WA, the age standardized rate for $\mathrm{CRC}$ is about 3-4-fold lower in SA, although the incidence appears to be increasing quite rapidly (Ibrahim et al., 2008), probably due to the adoption of a more Western diet. Little is known however about the incidence of LS in the Saudi population and to our knowledge there are no reflex MSI- or IHC-based screening programs to help detect LS in the routine clinical setting. A recent publication involving $807 \mathrm{CRC}$ cases from Riyadh reported an MSI frequency of $11.3 \%$ and LS frequency of $0.9 \%$ (Siraj et al., 2015). The LS frequency is similar to previous results from large Australian (Schofield et al., 2009; Ward et al., 2013), Spanish (Pinol et al., 2005) and American (Samowitz et al., 2001) studies, but lower than estimates of $5.1 \%$ (Zeinalian et al., 2015) and 2\% (Nemati et al., 2011) from Iranian studies. It should be noted however that the latter studies were based on clinical findings (Amsterdam criteria) rather than genetic findings.

In the present study we have retrospectively screened Saudi CRC cases from the Eastern Province and from
Riyadh for MSI and BRAF mutation as a first step in the identification of LS cases. The results are compared with those of a similar study conducted in the WA population (Schofield et al., 2009), which has a 3-fold higher incidence of CRC and a much older mean patient age compared to the Saudi population.

\section{Materials and Methods}

\section{Patient cohorts}

All patients with primary CRC diagnosed at three hospitals in Dammam ( $n=191$; King Fahad Specialist Hospital, King Fahad University Hospital, Dammam Regional Laboratory) and the King Khaled University Hospital in Riyadh (n=93) between 2006 and 2015 were eligible. Because the large majority of LS patients are diagnosed with CRC at a young age (Lynch et al., 2003 ), only patients aged $<60$ years were included. No information was available regarding the family history of cancer. Clinicopathological information including gender, age, stage, tumor site and histological grade were obtained from pathology records. For each case, sections of $10 \mu \mathrm{m}$ thickness (for DNA extraction) or $4 \mu \mathrm{m}$ thickness (for IHC) were cut from archival, paraffin-embedded tumor and normal tissue blocks were obtained from surgically resected specimens. Each block was verified for maximal tumor cell content $(>50 \%)$ by examination of $H \& E$ stained slides by a pathologist. The study was approved by the human research ethics committees of the King Fahad Specialist Hospital-Dammam (IRB LAB 055), King Khaled University Hospital (15/0148/IRB), King Fahad University Hospital (IRB-2014-01-297) and Dammam Regional Laboratory (approval date 18/08/2014).

\section{DNA extraction}

DNA extraction from paraffin-embedded tissue sections was performed using a kit and automated DNA extractor as described by the manufacturer (MagNA Pure Compact, Roche, USA). DNA purity and concentration were evaluated by NanoDrop spectrophotometer.

\section{MSI analysis}

Initial MSI screening was performed using PCR and fluorescent-single strand conformation polymorphism (F-SSCP) analysis to detect deletions in the BAT-26 mononucleotide repeat (Iacopetta et al., 2000). All positive cases were confirmed using the commercially available pentaplex MSI analysis system as described by the manufacturer (Promega, Australia). This contains five mononucleotide repeat markers (BAT-25, BAT-26, NR-21, NR-24 and MONO-27) that are highly susceptible to somatic deletions in tumors with defective MMR (Suraweera et al., 2002), as well as two pentanucleotide markers used to ensure correct sample matching. PCR products were run on an ABI 3500 genetic analyzer and the length of allelic deletions was quantified using Genemapper software (ABI, California, USA). Deletion lengths and frequencies were compared to those of MSI cases detected in young CRC patients in WA using the pentaplex system during routine evaluation. 


\section{$B R A F$ and KRAS mutation analysis}

Samples were screened for mutations in codon 600 of BRAF using competitive allele-specific Taqman (CAST)PCR (Life Technologies, USA) as described previously by our group (Richter et al., 2013). Data was collected during 40 cycles of amplification and analysed using the Mutation DetectorTM software v.2.0 (Life Technologies, USA). Samples with a $\Delta(\mathrm{d}) \mathrm{Ct}$ of less than 9.96 were considered positive for mutation, where $\Delta \mathrm{Ct}=\mathrm{Ct}$ mut $-\mathrm{Ct}$ ref.

Mutations in codon 600 of BRAF were confirmed using the Therascreen ${ }^{\circledR}$ BRAF Pyrosequencing Kit (Qiagen, Australia) according to manufacturer's instructions. The KRAS Pyrosequencing kit (Qiagen, Australia) was also used to detect mutations in codons 12, 13 and 61 of the KRAS gene. Pyrosequencing was performed on the PyroMark Q24 platform (Qiagen) using Therascreen buffers and reagents (v1). Readouts were generated with the PyroMark Q24 software (v. 2.0.6.) and data was analyzed manually or with a plug-in tool provided by Qiagen.

\section{Immunohistochemistry}

Tumor samples that were found to be positive for MSI and wildtype for BRAF were investigated for loss of expression of the MLH1, PMS2, MSH2 and MSH6 proteins using IHC as described previously (Schofield et al., 2009). Briefly, $4 \mu \mathrm{m}$ tissue sections were cut serially from the same tumor blocks used to provide tissue samples for DNA extraction. IHC for MLH1, PMS2, MSH2 and MSH6 expression was performed using commercially available antibodies (clones M1, EPR3947, G219-1129 and 44, respectively) at the recommended dilutions (Ventana, Australia). Normal colonic epithelium located adjacent to tumor cells served as the internal positive control for MMR protein expression. Tumors were scored as showing normal expression, partial loss of expression or complete loss of expression.
Statistical analysis

Comparison between SA and WA MSI cases for the length of allelic deletions was performed using the Student's t-test. Associations between the presence of MSI and clinicopathological features was evaluated using Fisher's exact test. Significance was assumed at $\mathrm{P}<0.05$.

\section{Results}

A total of 284 cases were successfully tested for MSI using PCR and F-SSCP analysis for deletions in the BAT-26 marker. Representative results obtained with this screening technique are shown in Figure 1. Thirty three cases (11.6\%) showed deletions in BAT-26 and all were confirmed as having MSI using the pentaplex system that analyzes 5 mononucleotide repeats (Figure 2). Associations between MSI and clinicopathological features are shown in Table 1. Younger and male patients showed a trend for higher MSI frequency. Right-sided tumors also showed a higher frequency of MSI compared

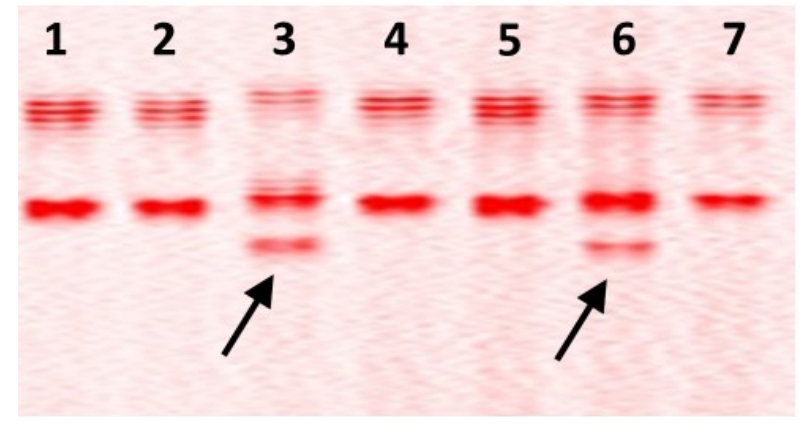

Figure 1.F-SSCP gel electrophoresis showing deletions in the mononucleotide repeat BAT-26 in two tumor samples from young Saudi CRC patients (arrows), indicating the presence of MSI.

Table 1. Clinicopathological Features of MSI Tumors in Young Saudi Arabian Colorectal Cancer Patients

\begin{tabular}{|c|c|c|c|c|c|c|c|}
\hline & Total & $\%$ & MSI & $\%$ & MSS & $\%$ & $\mathrm{P}$ \\
\hline Total no. of cases & 284 & & 33 & 11.6 & 251 & 88.4 & \\
\hline \multicolumn{8}{|l|}{ Age (years) } \\
\hline under 40 & 43 & 15 & 6 & 14 & 37 & 86.1 & \multirow[t]{3}{*}{0.37} \\
\hline $41-50$ & 88 & 31 & 13 & 14.8 & 75 & 85.2 & \\
\hline $51-60$ & 153 & 54 & 14 & 9.2 & 139 & 90.9 & \\
\hline \multicolumn{8}{|l|}{ Sex } \\
\hline Male & 146 & 51 & 20 & 13.7 & 126 & 86.3 & \multirow[t]{2}{*}{0.26} \\
\hline Female & 138 & 49 & 13 & 9.4 & 125 & 90.6 & \\
\hline \multicolumn{8}{|l|}{ Tumor Site* } \\
\hline Left Colon & 201 & 71 & 20 & 9.9 & 181 & 90.1 & \multirow[t]{2}{*}{0.13} \\
\hline Right Colon & 81 & 29 & 13 & 16 & 66 & 81.5 & \\
\hline \multicolumn{8}{|l|}{ Grade } \\
\hline Poorly differentiated & 18 & 6 & 2 & 11.1 & 16 & 88.9 & \multirow[t]{3}{*}{0.93} \\
\hline Mod. differentiated & 238 & 84 & 27 & 11.3 & 211 & 88.7 & \\
\hline Well differentiated & 28 & 10 & 4 & 14.3 & 24 & 88.7 & \\
\hline \multicolumn{8}{|l|}{ Stage $* *$} \\
\hline I & 9 & 5 & 0 & 0 & 9 & 100 & \multirow[t]{5}{*}{0.30} \\
\hline II & 122 & 65 & 12 & 9.8 & 110 & 90.2 & \\
\hline III & 25 & 13 & 4 & 16 & 21 & 84 & \\
\hline IV & 32 & 17 & 6 & 18.8 & 26 & 81.3 & \\
\hline Unknown & 96 & & 11 & 11.6 & 85 & 88.5 & \\
\hline
\end{tabular}

*Tumor site was not reported for 2 cases; ** Tumor site was not reported for 96 samples. 


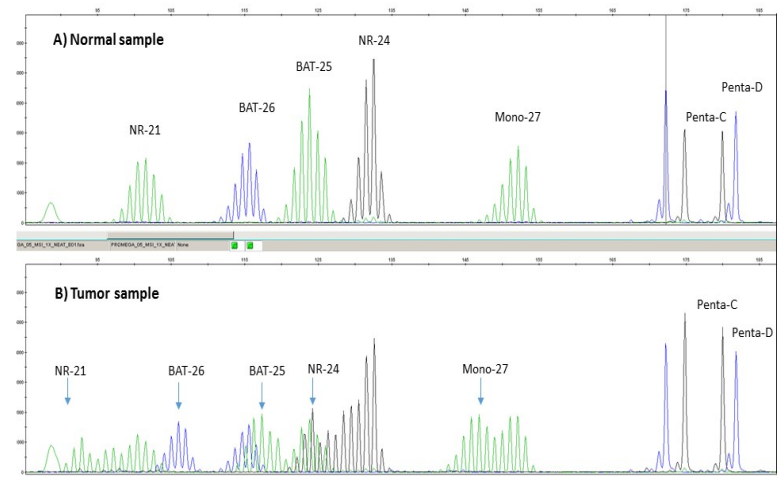

Figure 2. Capillary Electrophoresis (ABI 3500) Showing Results Obtained with the Pentaplex Panel of 5 Mononucleotide Repeat Markers (NR-24, BAT26, BAT-25, NR-24,MONO-27) Used to Confirm the Presence of MSI. The $\mathrm{x}$-axis is the PCR product size (bp) while the $y$-axis is the fluorescence intensity. Green, blue, and black peaks are amplification products from different microsatellite loci. Note the additional, shortened alleles (arrows) present in the tumor sample (B) compared to the normal mucosa sample (A), indicating MSI. Pentanucleotide markers shown on the right-hand side ensure correct sample matching of normal and tumor tissues

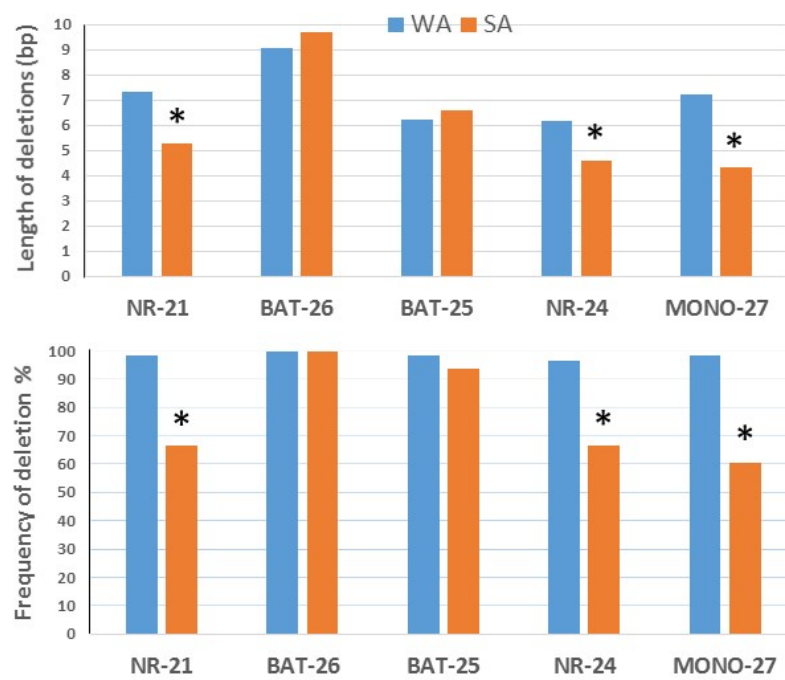

Figure 3. Average Lengths of Deletion in 5 Mononucleotide Repeat markers (NR-21, BAT-26, BAT-25, NR-24, MONO-27) was Compared between MSI Tumors from the WA and Saudi Populations. (A). The frequency of deletions in these markers was also compared (B). Statistically significant differences between the two populations are indicated by an asterix $(\mathrm{P}<0.05)$

to left-sided tumors, however this did not reach statistical significance $(\mathrm{P}=0.13$, Fisher's exact test).

The length and frequency of allelic deletions was quantified in each of the 5 mononucleotide repeats of the pentaplex assay. This analysis was performed in $33 \mathrm{MSI}$ cases from SA and in 56 age-matched MSI cases from WA (Figure 3). BAT-26 was deleted in all cases in both cohorts, with a similar average length of deletion $(9.1 \mathrm{bp}$ in WA and 9.7bp in SA). Interestingly, the average length of deletion for the NR-21, NR-24 and MONO-27 markers was significantly shorter in MSI tumors from SA (Figure $3 \mathrm{~A})$. This was due to the lower frequency of deletion of
A. BRAF mutation detection by Pyrosequencing

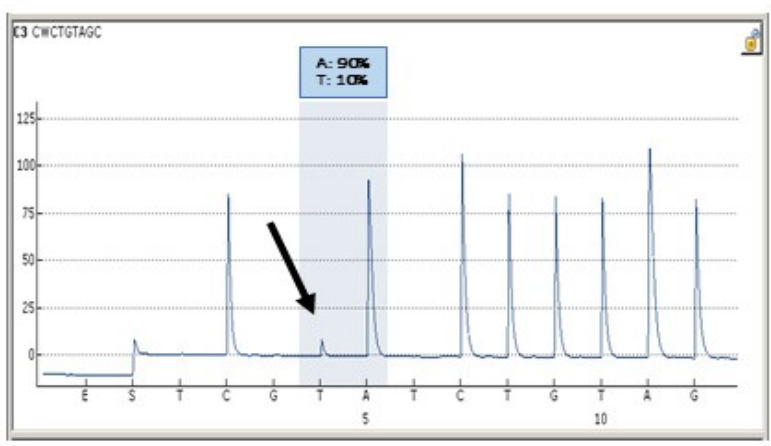

B. BRAF mutation detection by Sanger Sequencing
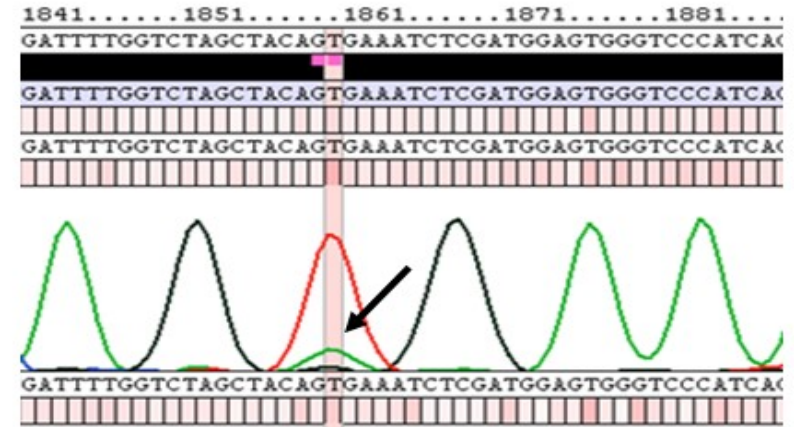

Figure 4. BRAF Mutation (Arrow) with Low Allele Frequency Shown Using Pyrosequencing. (A) and confirmed by Sanger sequencing (B). The mutation is C.1799T >A, p.Val600Glu in exon 15

A. KRAS mutation detection by F-SSCP
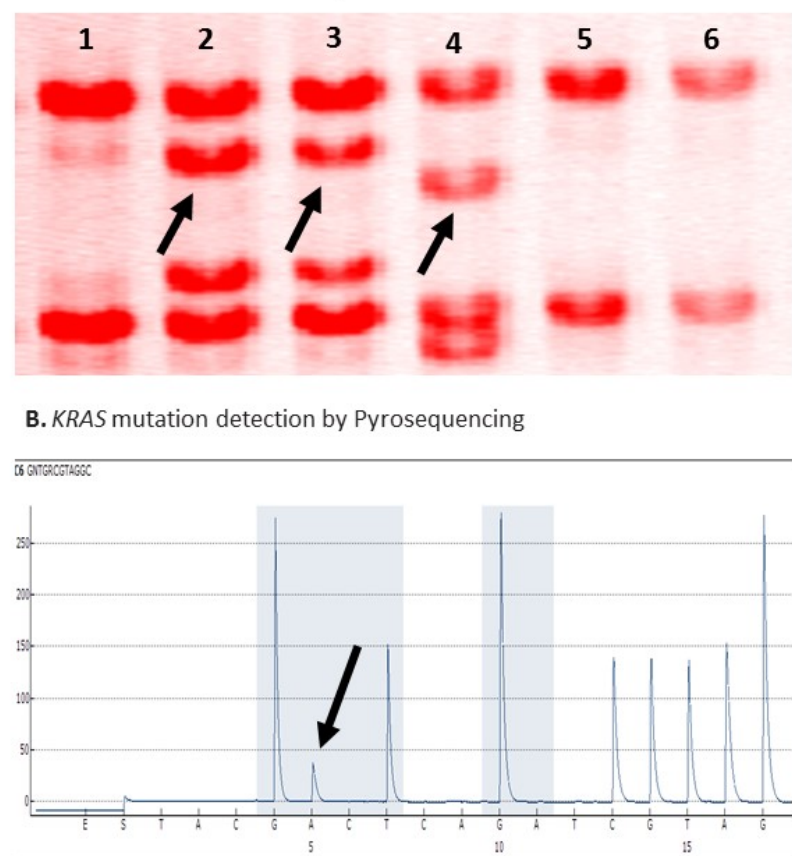

Figure 5. KRAS Mutations in Codons 12 and 13 Revealed by F-SSCP. (A) and confirmed by pyrosequencing (B). The mutation shown by pyrosequencing is c. $35 \mathrm{G}>\mathrm{A}$, p.Gly12Asp

these 3 markers in MSI tumors from SA (Figure 3B). Nine of the 33 MSI tumors from SA (27.3\%) showed deletions only in the BAT-26 and BAT-25 markers.

We next screened the 33 MSI cases from SA for 


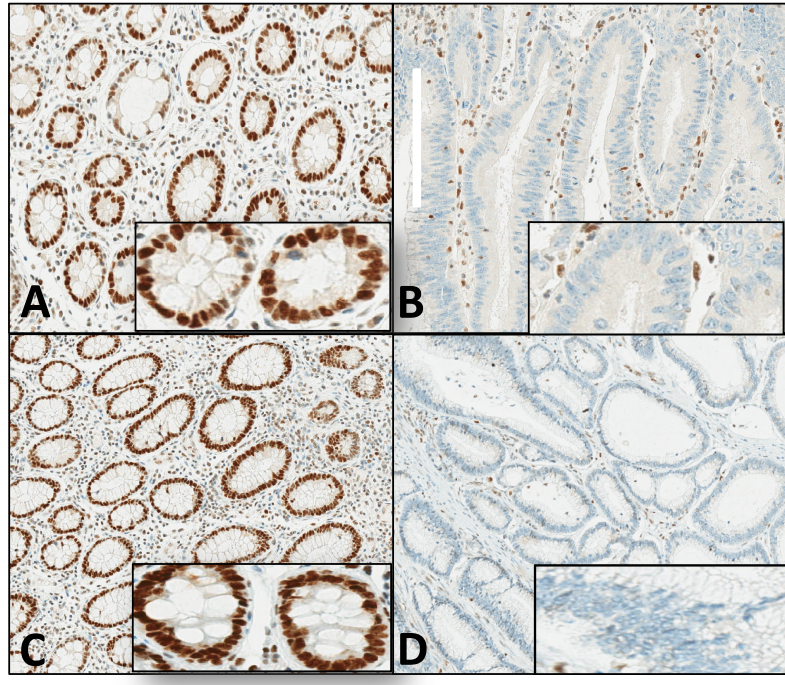

Figure 6. Immunohistochemical Staining for the Mismatch Repair Proteins MSH2 (A and B) and MLH1 (C and D). A and C show areas of positive staining in adjacent normal mucosa, while $\mathrm{B}$ and $\mathrm{D}$ show complete lack of staining of the respective MMR protein. Magnification: 200x and 500x (inset)

BRAF mutations using CAST real time PCR. Only one tumor was found to have a BRAF mutation and this was confirmed using pyrosequencing (Figure 4A). The mutant allele frequency was estimated at just 5-10\% and this was independently confirmed using Sanger DNA sequencing (Figure 4B). Therefore, all MSI cases in this series except one were possible candidates for LS.

The incidence of KRAS mutation amongst the MSI cases was determined by initial screening with F-SSCP followed by pyrosequencing to confirm and identify mutations (Figure 5). The observed KRAS mutation frequency was $27.3 \%(9 / 33)$.

Loss of expression of the four major MMR proteins (MLH1, PMS2, MSH2, MSH6) was examined by IHC in 30 MSI cases for which tissue was available. Representative results for IHC are shown in Figure 6. Seven cases showed loss of both MSH2 and MSH6, 9 showed loss of both MLH1 and PMS2, 11 showed loss of PMS2 only, while 3 showed apparently normal staining with no loss of expression for any of the MMR proteins. Tissue was not available for MLH1 analysis in 3 of the 11 tumors that showed loss of PMS2 only.

\section{Discussion}

The incidence of CRC in the Saudi population is increasing rapidly and is currently the most frequent cancer type in males (Ibrahim et al., 2008; Al-Madouj et al., 2011). The average age of Saudi CRC patients at diagnosis is approximately 15 years younger than Western CRC patients (55 vs 70 years, respectively) (Aljebreen, 2007; Mosli et al., 2012; Amin et al., 2012). Although the incidence of consanguinity in SA is over $50 \%$ (El-Hazmi et al., 1995), it is unclear whether this contributes to a higher incidence of familial cancer syndromes. The aim of this work was therefore to establish whether MSI screening of young CRC patients ( $<60$ years) could help to identify unrecognized cases of LS in SA. Screening for MSI was performed in the absence of information on the family history of cancer in these patients. A similar approach used in a Western population was shown to increase the detection rate of LS in young CRC patients 3-fold and this strategy has now been adopted as routine clinical practice in WA (Schofield et al., 2009; Schofield et al., 2014).

Somatic deletion in the BAT-26 mononucleotide repeat is a sensitive marker for MSI in CRC (Iacopetta et al., 2010). Using a rapid F-SSCP technique (Figure 1), MSI was found in $11.6 \%(33 / 284)$ of young CRC patients from the Eastern province of Dammam and from Riyadh. This frequency is almost identical to that reported (11.3\%) in an unselected cohort of 807 CRC patients from Riyadh (Siraj et al., 2015). In comparison, the MSI frequency found in young CRC patients from WA was significantly lower (7.8\%, 105/1,344, P=0.045) (Schofield et al., 2009). The higher frequency of MSI observed in young patients from SA may reflect a higher prevalence of LS compared to Western populations, however this awaits germline testing of the $32 \mathrm{MSI} / \mathrm{BRAF}$ wildtype cases found here. The anatomical distribution of MSI cases was also different. In WA, the majority of MSI tumors from young patients arise in the proximal colon $(90 / 118,76 \%$ ) (Schofield et al., 2009). In contrast, less than half of the MSI tumors were right-sided in the current study $(13 / 33,39 \%$; $\mathrm{P}<0.001)$ and in another study of unselected Saudi CRC patients (38/90, 42\%) (Siraj et al., 2015). These results suggest there may be differences in the etiology and pathogenesis of MSI tumors between Saudi and Western populations.

The pentaplex system for evaluating MSI status (Suraweera et al., 2002) is considered superior to the Bethesda panel that includes both dinucleotide and mononucleotide repeat markers (Xicola et al., 2007). We used this system to confirm the MSI status of all 33 cases identified by F-SSCP screening with BAT-26 (Figure 2). For each of the 5 mononucleotide repeats we also compared the average length of allelic deletion and the frequency of allelic deletion between SA and WA patients. While BAT-25 and BAT-26 showed a similar pattern of somatic deletion between the two cohorts, the NR-21, NR24 and MONO-27 markers were deleted less frequently (Figure 3B), thus accounting for the shorter average length of deletions (Figure 3A). A sizeable minority of the Saudi MSI cases $(9 / 33,27 \%)$ showed no deletions in any of the NR-21, NR-24 and MONO-27 markers, whereas these were each deleted in at least $95 \%$ of MSI cases from WA. To our knowledge, such geographic differences in the frequency of allelic deletion have not been previously reported for MSI tumors. Our findings confirm that BAT26 is an excellent marker for MSI screening because the large size of deletions (average of 9-10 bp in WA and SA $\mathrm{CRC}$ ) facilitate detection.

The presence of BRAF mutation is used to distinguish MSI tumors that are sporadic in origin from those that may be LS (Domingo et al., 2004). Of the 33 MSI cases identified in this cohort of young Saudi CRC patients, only $1(3 \%)$ contained a BRAF mutation, therefore excluding it as a potential LS case. A very low frequency of BRAF mutation $(2.5 \%, 19 / 757)$ was also reported by Siraj et al (2014) in an unselected cohort of Saudi CRC 
patients, whereas a higher BRAF mutation frequency (7/105, 6.7\%) was reported for MSI cases from young Australian CRC patients (Schofield et al., 2009). The single BRAF mutation detected here showed a very low allelic frequency (Figure 4), suggesting the presence of tumor heterogeneity. The remaining 32 MSI cases with wildtype BRAF are candidates for germline mutation in MMR genes as a cause of their MSI phenotype.

The presence of BRAF mutation in CRC is mutually exclusive with that of KRAS mutation (Li et al., 2006). Because of the low BRAF mutation frequency observed here and in a previous study of Saudi CRC (Siraj et al., 2015), we investigated whether this was compensated by a higher frequency of KRAS mutation. The KRAS mutation frequency observed here $(27 \%, 9 / 33)$ was similar to that reported in a study of unselected Saudi CRC patients (30\%) (Beg et al., 2015), but less than reported in a study of young Saudi CRC patients (40\%) (Elsamany et al., 2014). We are not aware of any reports that have investigated the frequency of KRAS mutation specifically in MSI CRC tumors.

MSI is almost always accompanied by loss of expression of one or more MMR proteins, usually as MLH1/PMS2 or MSH2/MSH6 combinations. This information is important for directing germline mutation analysis to the appropriate gene(s). In the present study, all but 3 of the 30 MSI cases examined by IHC showed loss of expression of MMR proteins. In our earlier study, 2 of 97 MSI cases showed no apparent loss of expression (Schofield et al., 2009), while a Saudi study also found discordant IHC results in 2 of 73 MSI tumors (Siraj et al., 2015). Such rare discordant cases may be explained by failure of the MMR gene mutation to alter protein antigenicity, or by mutation of a different MMR gene family member as the cause of MSI. In the present study we found a similar proportion of MLH1/PMS2 and MSH2/ MSH6 double loss cases compared to Australian MSI cases (9 and 7 vs 42 and 30, respectively) (Schofield et al., 2009), but a higher percentage with loss of PMS2 only ( $37 \%$ vs $7 \%$, respectively). Tissue was not available in 3 of the 11 cases with PMS2 loss to test for concomitant loss of MLH1. Nevertheless, this result suggests that PMS2 mutations may be more frequent in the Saudi population.

In conclusion, we have shown that screening with the BAT-26 mononucleotide repeat marker is an efficient way to detect MSI in archival tumor samples from Saudi CRC patients. The MSI frequency was significantly higher compared to a similar age cohort of Australian CRC patients. Analysis of results obtained with 5 mononucleotide repeat markers revealed novel differences in allelic deletions between MSI tumors from Saudi and Australian patients. The anatomic distribution of MSI cases was also markedly different, with a more even distribution of MSI cases between the left and right colon in Saudi patients. Our results confirm the low frequency of BRAF mutations in MSI tumors reported earlier in unselected Saudi CRC patients. This marker allows exclusion of rare MSI/BRAF mutant patients from further follow up as possible LS cases.

The next step in this work is to follow up surviving
MSI patients and to perform germline mutation analysis of MMR genes in individuals who give consent for genetic testing and following appropriate pre-test counselling. The IHC results should help to inform this analysis. The incidence of LS amongst Saudi CRC patients was recently estimated to be approximately $1 \%(8 / 807)$ (Siraj et al., 2015). This is similar to the incidence of $0.8-1 \%$ reported in CRC cohorts from Western populations (Samowitz et al., 2001; Pinol et al., 2005; Schofield et al., 2009; Ward et al., 2013).The present study may help to justify the introduction of routine MSI screening of young CRC patients as a cost-effective way (Snowsill et al., 2015) to identify LS in the Saudi population.

\section{Acknowledgements}

The authors would like to thank Dr Dalal Altamimi, Dr Mohamed El-Shawarby, Dr Ramez Atassi, Ahmed Alotaibi, Saber Alzahrani, Clara Kutti, Edison Tan, Abdulrahman Hazzazi, Hala Almadani and Shaker Ahmed for their technical support and provision of clinical data. We are also grateful to the Anatomical Pathology Department at King Fahad University Hospital, Dammam University, the Histopathology Department at Dammam Regional Laboratory \& Blood Bank, Ministry of Health, and the Department of Pathology and Laboratory Medicine, King Fahad Specialist Hospital-Dammam.

\section{References}

Aljebreen AM (2007). Clinico-pathological patterns of colorectal cancer in Saudi Arabia: younger with an advanced stage presentation. Saudi J Gastroenterol, 13, 84-87.

Al-Madouj A, Hayder M, Al-Zahrani W, et al (2011). Cancer incidence report saudi arabia. Saudi Cancer Registry 2011. http://www.chs.gov.sa/Ar/HealthRecords/CancerRegistry/ Pages/CancerRegistryRecords.aspx

Amin TT, Suleman W, Al Taissan AA, et al (2012). Patients' profile, clinical presentations and histopathological features of colo-rectal cancer in Al Hassa region, Saudi Arabia. Asian Pac J Cancer Prev, 13, 211-6.

Beg S, SirajAK, Prabhakaran S, et al (2015). Molecular markers and pathway analysis of colorectal carcinoma in the Middle East. Cancer. 121, 3799-808.

Domingo E, Laiho P, Ollikainen M, et al (2004). BRAF screening as a low-cost effective strategy for simplifying HNPCC genetic testing. J Med Genet, 41, 664-8.

el-Hazmi MA, al-Swailem AR, Warsy AS, et al (1995). Consanguinity among the Saudi Arabian population. J Med Genet, 32, 623-26.

Elsamany SA, Alzahrani AS, Mohamed MM, et al (2014). Clinico-pathological patterns and survival outcome of colorectal cancer in young patients: western Saudi Arabia experience. Asian Pac J Cancer Prev, 15, 5239-43.

Iacopetta B, Grieu F (2000). Routine detection of the replication error phenotype in clinical tumor specimens using fluorescence-SSCP. Biotechniques, 28, 566-70.

Iacopetta B, Grieu F, Amanuel B (2010). Microsatellite instability in colorectal cancer. Asia Pac J Clin Oncol, 6, 260-9.

Ibrahim EM, Zeeneldin AA, El-Khodary TR, et al (2008). Past, present and future of colorectal cancer in the Kingdom of Saudi Arabia. Saudi J Gastroenterol, 14, 178-82.

Järvinen HJ, Aarnio M, Mustonen H, et al (2000). Controlled 15year trial on screening for colorectal cancer in families with 
hereditary nonpolyposis colorectal cancer. Gastroenterol, 118, 829-34.

Li WQ, Kawakami K, Ruszkiewicz A, et al (2006). BRAF mutations are associated with distinctive clinical, pathological and molecular features of colorectal cancer independently of microsatellite instability status. Mol Cancer, $\mathbf{5}, 2$.

Lynch HT, de la Chapelle A (2003). Hereditary colorectal cancer. N Engl J Med, 348, 919-32.

Lynch HT, Riley BD, Weissman SM, et al (2004). Hereditary nonpolyposis colorectal carcinoma (HNPCC) and HNPCClike families: Problems in diagnosis, surveillance, and management. Cancer, 100, 53-64.

Marcus VA, Madlensky L, Gryfe R, et al (1999). Immunohistochemistry for hMLH1 and hMSH2: a practical test for DNA mismatch repair-deficient tumors. Am J Surg Pathol, 23, 1248-55.

Mosli MH, Al-Ahwal MS (2012). Colorectal cancer in the Kingdom of Saudi Arabia: need for screening. Asian Pac J Cancer Prev, 13, 3809-13.

Nemati A, Rahmatabadi ZK, Fatemi A, Emami MH (2011). Hereditary non-polyposis colorectal cancer and familial colorectal cancer in central part of Iran, Isfahan. J Res Med Scien, 17, 67-73.

Piñol V, Castells A, Andreu M, et al (2005). Gastrointestinal oncology group of the spanish gastroenterological association. accuracy of revised bethesda guidelines, microsatellite instability, and immunohistochemistry for the identification of patients with hereditary nonpolyposis colorectal cancer. JAMA, 293, 1986-94.

Richter A, Grieu F, Carrello A, et al (2013). A multisite blinded study for the detection of BRAF mutations in formalin-fixed, paraffin-embedded malignant melanoma. Sci Rep, 3, 1659.

Samowitz WS, Curtin K, Lin HH, et al (2001). The colon cancer burden of genetically defined hereditary nonpolyposis colon cancer. Gastroenterol, 121, 830-8.

Schofield L, Watson N, Grieu F, et al (2009). Population-based detection of Lynch syndrome in young colorectal cancer patients using microsatellite instability as the initial test. Int J Cancer, 124, 1097-102.

Schofield L, Grieu F, Amanuel B, et al (2014). Population-based screening for Lynch syndrome in Western Australia. Int $J$ Cancer, 135, 1085-91.

Siraj AK, Bu R, Prabhakaran S, et al (2014). A very low incidence of BRAF mutations in Middle Eastern colorectal carcinoma. Mol Cancer, 13, 168.

Siraj AK, Prabhakaran S, Bavi P, et al (2015). Prevalence of Lynch syndrome in a Middle Eastern population with colorectal cancer. Cancer, 121, 1762-71.

Snowsill T, Huxley N, Hoyle M, et al (2015). A model-based assessment of the cost-utility of strategies to identify Lynch syndrome in early-onset colorectal cancer patients. $B M C$ Cancer, 15, 313 .

Suraweera N, Duval A, Reperant M, et al (2002). Evaluation of tumor microsatellite instability using five quasimonomorphic mononucleotide repeats and pentaplex PCR. Gastroenterolo, 123, 1804-11.

Terdiman JP (2005). It is time to get serious about diagnosing Lynch syndrome (hereditary nonpolyposis colorectal cancer with defective DNA mismatch repair) in the general population. Gastroenterol, 129, 741-4.

Umar A, Boland CR, Terdiman JP, et al (2004). Revised Bethesda Guidelines for hereditary nonpolyposis colorectal cancer (Lynch syndrome) and microsatellite instability. $J$ Natl Cancer Inst, 96, 261-8.

Ward RL, Hicks S, Hawkins NJ (2013). Population-based molecular screening for Lynch syndrome: implications for personalized medicine. J Clin Oncol, 31, 2554-2562.
Xicola RM, Llor X, Pons E, et al (2007). Gastrointestinal oncology group of the spanish gastroenterological association. performance of different microsatellite marker panels for detection of mismatch repair-deficient colorectal tumors. J Natl Cancer Inst, 99, 244-52.

Zeinalian M, Hashemzadeh-Chaleshtori M, Akbarpour MJ, et al (2015). Epidemioclinical feature of early-onset colorectal cancer at-risk for Lynch syndrome in central Iran. Asian Pac J Cancer Prev, 16, 4647-52. 